\title{
Uso no-convencional del mucílago de avena en fotoprotección
}

\section{Unconventional use of oat mucilage in photoprotection}

\author{
RAMÍREZ-GRANADOS, Juan Carlos $\dagger^{*}$, DÍAZ-PÉREZ, César, ALMANZA-GUERRERO, Lisbeth y \\ ENRÍQUEZ-ARREDONDO, María José
} Universidad de Guanajuato, División de Ciencias de la Salud e Ingenierías, Departamento de Ingeniería Agroindustrial.
Prolongación Río Lerma s/n. Col. Suiza, Celaya, Gto., México. C.P. 38060

ID $1^{\mathrm{er}}$ Autor: Juan Carlos Ramírez-Granados / ORC ID: 0000-0001-6460-6472, Researcher ID Thomson: S-5874-2018, CVU CONACYT ID: 167866

ID $1^{\mathrm{er}}$ Coautor: César, Díaz-Pérez / CVU CONACYT ID: 101579

ID $2^{\text {do }}$ Coautor: Lisbeth, Almanza-Guerrero / ORC ID: 0000-0003-2468-2424

ID $3^{\text {er }}$ Coautor: María José, Enríquez-Arredondo / ORC ID: 0000-0002-8310-1397

DOI: $10.35429 / J I D .2019 .8 .3 .10 .16$

Recibido 09 Julio, 2019; Aceptado 03 de Septiembre, 2019

\begin{abstract}
Resumen
En este trabajo se estudiaron algunas características espectrales del mucílago de avena para obtener un conocimiento profundo y detallado acerca de la posible aplicación de este material en la absorción de radiación ultravioleta peligrosa para la piel humana. Para este propósito se extrajo y purificó el mucílago de avena adaptando un método previamente reportado. Luego, fueron preparadas varias disoluciones acuosas con diferentes concentraciones conocidas de este extracto. Se analizaron estas disoluciones con un espectrofotómetro para determinar su absorbancia en el rango de 200 a 1000 $\mathrm{nm}$. A través de los espectros de absorbancia se evaluó la capacidad fotoprotectora del mucílago de avena en disolución acuosa. También se calculó el coeficiente de extinción de este material a través de las mediciones espectrofotométricas y la Ley de Lambert-Beer. Además, se elaboró una crema con ingredientes naturales y mucílago de avena como compuesto activo para evaluar su factor de protección solar y compararlo con el del mucílago acuoso. Finalmente, se analizó la factibilidad de utilizar el mucílago de avena como ingrediente activo en protectores solares efectivos y seguros para las personas y el ambiente.
\end{abstract}

Protección solar, Mucílago, Avena

\begin{abstract}
In this work, some spectral characteristics of oat mucilage were studied to obtain a deep and detailed knowledge about the possible application of this material in the absorption of dangerous ultraviolet radiation for human skin. For this purpose, the mucilage of oat was extracted and purified by adapting a previously reported method. Then, several aqueous solutions with different known concentrations of this extract were prepared. These solutions were analyzed with a spectrophotometer to determine their absorbance between 200 and $1000 \mathrm{~nm}$. Through the absorbance spectra, the photoprotective capacity of the mucilage of oat in aqueous solution was evaluated. The extinction coefficient of this material was also calculated through spectrophotometric measurements and the Lambert-Beer Law. In addition, a cream with natural ingredients and oat mucilage as active compound was prepared to evaluate its sun protection factor and compare it with that of the aqueous mucilage. Finally, the feasibility of using the mucilage of oat as active ingredient in effective and safe sunscreens for people and the environment was analyzed.
\end{abstract}

Sun protection, Mucilage, Oat

Citación: RAMÍREZ-GRANADOS, Juan Carlos, DÍAZ-PÉREZ, César, ALMANZA-GUERRERO, Lisbeth y ENRÍQUEZARREDONDO, María José. Uso no-convencional del mucílago de avena en fotoprotección. Revista del Diseño Innovativo. 2019. 3-8: 10-16

\footnotetext{
* Correspondencia del Autor (Correo electrónico: jcramirez@ugto.mx)

$\dagger$ Investigador contribuyendo como primer autor.
} 


\section{Introducción}

El Sol es necesario para el ser humano en muchos aspectos importantes, uno de ellos es la síntesis de vitamina D. Sin embargo, se deben tener precauciones porque en exceso los rayos solares pueden ser dañinos para la piel. Dentro del espectro ultravioleta (UV) emitido por el Sol, el componente que encierra más peligro es el UVB, por tener la capacidad de dañar las cadenas de ADN. Actualmente se considera que estas radiaciones son culpables del $90 \%$ de los cánceres de piel, incluido el melanoma, cuya mortalidad supera la del resto de las neoplasias dérmicas. (Castañeda, 2016).

Los dos tipos más comunes de cáncer de piel son el melanoma y el no-melanoma. Los cánceres de piel tipo no-melanoma, se clasifican como carcinomas de células basales y carcinomas de células escamosas. El melanoma, tiene una tasa de mortalidad muy alta, ocupa el segundo lugar después de la leucemia en términos de años perdidos de vida potencial por muerte desde la edad promedio. (Hellwig, 2012).

La notable incidencia de los cánceres de piel y los efectos del fotodaño causados por la radiación ultravioleta ha aumentado el uso de agentes de protección solar, que han demostrado efectos beneficiosos para reducir los síntomas y la recurrencia de estos problemas. (Latha, 2013). El uso de protector solar mantiene a las personas seguras del Sol mientras previenen los cánceres de piel y el envejecimiento prematuro.

Aunque se consideran seguros, los agentes de protección solar no están libres de efectos adversos, el uso prolongado e inadecuado de filtros solares de origen químico (la mayoría de los filtros solares comerciales) puede generar efectos secundarios como una lenta pero progresiva intoxicación del organismo humano debido a que la piel absorbe los compuestos químicos, generando reacciones fotoalérgicas e inhibiendo la síntesis de vitamina D. Es por ello que se pretende mejorar la comprensión de los consumidores sobre los productos de protección solar, al mismo tiempo que se enfrenta el desafío de buscar materiales orgánicos que puedan ser utilizados en la fabricación de filtros solares más saludables para las personas y amigables con el medio ambiente. El material de origen natural que se seleccionó en este trabajo fue el mucílago de avena debido a su inocuidad, alta disponibilidad, bajo costo de producción y relativa sencillez de extracción.
La eficacia de un filtro solar se prueba in vitro o in vivo a través del factor de protección solar (FPS), índices UVA y su perfil de protección UV. El FPS es el método aceptado para evaluar la protección relativa del protector solar contra las quemaduras solares. Se calcula dividiendo la cantidad de radiación UV requerida para producir un eritema mínimo en la piel en la que se ha aplicado un producto de protección solar, entre la cantidad de radiación UV requerida para producir un eritema mínimo en la piel desprotegida.

Los productos con FPS alto brindan más protección contra los efectos peligrosos de la luz solar que aquellos con FPS bajo, sin embargo, esto no quiere decir que se pueda permanecer más tiempo en exposición al Sol. La capacidad de un filtro solar para absorber la radiación UV se mide en términos del valor del coeficiente de extinción $(\varepsilon)$. Organismos reguladores como la FDA (Federal Drug Administration) exige que todos los filtros solares contengan una etiqueta indicando el factor de protección solar. (Cázares, 2013).

Las substancias fotoprotectoras son elaboradas como emulsiones del tipo aceite/agua o geles que idealmente no provocan daños o perjuicios a la salud. La idea de producir una crema de protección solar con mucílago de avena como ingrediente activo acompaña a la necesidad de nuevos productos para la protección contra la radiación UV y sigue la tendencia mundial de elaborar productos a partir de componentes naturales.

\section{Marco teórico}

La radiación ultravioleta es un tipo de luz invisible emitida por el Sol que consiste en longitudes de onda entre 200 y $400 \mathrm{~nm}$. La radiación UV se separa en tres componentes: UVA, UVB y UVC. Las longitudes de onda de 200 a 290 constituyen la radiación UVC, estos rayos no penetran nuestra atmósfera por lo que no son normalmente una causa de cáncer de piel; las longitudes de 290 a $320 \mathrm{~nm}$ constituyen radiación UVB y son la principal causa de las quemaduras por el Sol; mientras que las de 320 a $400 \mathrm{~nm}$ constituyen radiación UVA, estas penetran en la piel más profundamente, y se asocian con las arrugas, la pérdida de la elasticidad, fotoenvejecimiento, cáncer de piel, daños en el ADN nuclear y mitocondrial, y mutaciones genéticas.

RAMÍREZ-GRANADOS, Juan Carlos, DÍAZ-PÉREZ, César, ALMANZA-GUERRERO, Lisbeth y ENRÍQUEZ-ARREDONDO, María José. Uso no-convencional del mucílago de avena en fotoprotección. Revista del Diseño Innovativo. 2019 
La exposición a la radiación UVA generalmente permanece constante, mientras que la exposición a UVB ocurre más en el verano. La quemadura solar es el daño de la piel más comúnmente causado por la luz natural. (Hellwig, 2012). En América Latina, las personas han abandonado en gran medida la práctica de usar sombreros y ropas que brinden protección a la piel. Por lo tanto, la aplicación de protector solar se ha vuelto esencial para protegerse contra el aumento de la exposición al Sol. Los protectores solares son agentes que ayudan a prevenir que los rayos UV lleguen a la piel y consisten en formulaciones que bloquean los rayos UV y deben contener ciertas cantidades de sustancias que absorban dichos rayos.

El protector ideal sería aquella sustancia que opaca a ambas radiaciones UVA y UVB, biológicamente inerte, químicamente inalterable y aceptable cosmética y dermatológicamente. El uso y la aplicación inadecuada del protector solar también contribuyen al aumento de las quemaduras solares, a pesar de su uso frecuente. (Latha, 2013). La fotoprotección tópica moderna involucra factores protectores primarios (filtros solares) que absorben o reflejan la radiación UV y factores secundarios (antioxidantes, osmolitos y enzimas) que pueden interrumpir la cascada fotoquímica provocada por la penetración de los rayos UV, lo que limita el daño a la piel.

Los protectores solares de grado médico brindan suficiente protección solar, pero el requisito para el uso regular supone una carga financiera para el paciente que a menudo no es sostenible. Se han desarrollado varios filtros solares de venta libre en forma de cremas, lociones, geles, pomadas, aceites, barras $\mathrm{y}$ aerosoles a precios accesibles y están disponibles para su compra en lugares convenientes, como las tiendas locales. En los últimos tiempos, los protectores solares se comercializan como formulaciones cosméticas multifuncionales incorporadas a otros cosméticos como cremas hidratantes, bases faciales y bases de espuma. (Castanedo, 2014).

Algunos productos derivados de las plantas se pueden utilizar como filtros solares pues tienen la capacidad de absorber diferentes radiaciones. Normalmente, se formulan asociados a otros componentes de síntesis y/o pigmentos minerales para asegurar la eficacia filtrante del fotoprotector.
Estos extractos vegetales deben cumplir las características del filtro solar, es decir, han de presentar una absorción de la radiación que oscile entre 290 y $400 \mathrm{~nm}$ y han de ser estables en condiciones de uso y fabricación normales. Los mucílagos son un tipo de fibra soluble que poseen numerosas propiedades medicinales. Se los utiliza para combatir el estreñimiento, adelgazar, reducir los niveles sanguíneos de colesterol y efectos probióticos. Además, usados externamente los mucílagos tienen propiedades emolientes, es decir, hidratan y protegen la piel. Por este motivo pueden usarse para aliviar afecciones como heridas, grietas, ulceras, cortes, quemaduras, etc. Se encuentran de forma natural en algunas semillas, plantas y cereales.

La avena pertenece a la familia de las gramíneas, al igual que el resto de los cereales comestibles; una de las familias más importantes para la alimentación humana y cuyos frutos son granos secos y ricos en almidón, proteínas y mucílago. Así mismo contiene minerales como fósforo, potasio, magnesio, calcio y hierro. En el proceso de transformación de los granos en hojuelas no se elimina nada, sólo se pasan por cilindros para que queden aplastados. Esta es la mejor forma de aprovechar sus propiedades nutritivas. (Bizkaia, 2006). En el presente estudio el mucílago de avena fue investigado con el objetivo de analizar sus características espectrales y evaluar su acción fotoprotectora a través de la producción de una crema elaborada con una emulsión aceite/agua y con el mucílago de avena como sustancia activa. La evaluación del producto desarrollado se realizó mediante un método in vitro para la determinación de su factor de protección solar que fue reportado previamente (Mansur et al. 1986).

\section{Métodos y materiales}

\section{Extracción de mucílago de avena}

Para realizar la extracción del mucílago de avena se utilizaron hojuelas de avena marca Quaker® por su calidad y estándares de elaboración, y así asegurar que no se tuvieran contaminantes en la avena. Se tamizaron las hojuelas de avena para eliminar las partículas de polvo. Luego se pesaron $100 \mathrm{~g}$ del cereal con una balanza analítica. Se hidrató la avena con agua destilada en una proporción 1:7 p/v, aplicando agitación mecánica a $350 \mathrm{rpm}$ durante 16 horas. Posteriormente la alícuota se colocó dentro de un refrigerador durante 6 horas a $10^{\circ} \mathrm{C}$. 
Para la obtención del sobrenadante se realizó un doble filtrado, primero por decantación para la eliminación de partículas precipitadas y luego con tela de gasa para remover fragmentos de hojuelas de avena (dichos residuos fueron eliminados). Después se hizo un segundo filtrado con el fin de eliminar las partículas de avena más pequeñas que se quedaron aún en el sobrenadante, donde se utilizó papel filtro y una bomba de vacío. Luego, se calentó el sobrenadante a $75{ }^{\circ} \mathrm{C}$ durante 8 horas, monitoreando continuamente la temperatura para que se mantuviera constante. Una vez terminado el calentamiento se dejó enfriar a temperatura ambiente.

Para la precipitación del mucílago acuoso se agregó etanol en una relación 1:3 v/v (alícuota/etanol) y se dejó reposar una hora para su completa precipitación. A continuación, se realizó una tercera etapa de filtración para separar el mucílago precipitado de la fase etílica mediante papel filtro y con ayuda de una bomba de vacío (la fase con etanol fue desechada). Se eliminó el resto del solvente en el mucílago precipitado mediante su calentamiento en un horno a $70{ }^{\circ} \mathrm{C}$ durante 7 horas. Al estar el mucílago seco, se molió en un mortero para obtener un polvo seco. Se pesó el mucílago obtenido con una balanza analítica, se etiquetó y se almacenó a temperatura ambiente en un vaso de precipitados cubierto con una película de plástico para evitar que se contamine.

\section{Caracterización del mucílago de avena}

Se realizaron mediciones del espectro de absorbancia entre 200 y $1000 \mathrm{~nm}$ en intervalos de $10 \mathrm{~nm}$ con un espectrofotómetro UV-Visible Jenway 7305 a una disolución con mucílago de avena con concentraciones de $1 \mathrm{~g} / \mathrm{L}$; también se midió la absorbancia de $30 \mathrm{~mL}$ de la alícuota con mucílago de avena antes de la precipitación con etanol.

Se hizo también la determinación del coeficiente de extinción $(\varepsilon)$ mediante la ley de Lambert-Beer y mediciones de absorbancia. Esta ley expresa la relación entre absorbancia de la radiación monocromática (de longitud de onda única) y la concentración de un cromóforo en solución. El coeficiente de extinción es específico para cada cromóforo, por lo que para el mucílago de avena se calculó midiendo la absorbancia de 10 diluciones con concentraciones decrecientes conocidas que comenzaron en $1 \mathrm{~g} / \mathrm{L}$ y a $230 \mathrm{~nm}$.
Con los resultados obtenidos se realizó una regresión lineal para obtener la pendiente de la recta que mejor se ajusta a las mediciones. La pendiente de la recta de tendencia se relaciona con el coeficiente de extinción $\varepsilon$ a través de la siguiente ecuación:

$$
A=\log _{10}\left(\frac{I}{I_{0}}\right)
$$

La absorbancia $A$ esta definida como el logaritmo base 10 de las intensidades de entrada $\left(I_{0}\right)$ y salida $(I)$ en la muestra. La absorbancia también puede definirse a través de la Ley de Lambert-Beer como:

$A=\varepsilon c l$

donde $\varepsilon$ es el coeficiente de extinción, la cual es una constante a una determinada longitud de onda, $c$ es la concentración $(\mathrm{g} / \mathrm{L})$ y $l$ el ancho de la celda $(\mathrm{cm})$.

Para calcular el coeficiente de extinción $(\varepsilon)$, se divide la absorbancia entre la concentración de la molécula y el ancho de la muestra:

$\varepsilon=\frac{A}{c l}$

Las ecuaciones 2 y 3 corresponden a funciones lineales; por lo tanto, se espera que al graficarlas formen una recta. Para la elaboración de la crema con mucílago de avena se realizó una emulsión. Se pesó con una balanza analítica 1.5 $\mathrm{g}$ de cera de abeja, $1.5 \mathrm{~g}$ de aceite de coco y 1.5 $\mathrm{g}$ de manteca de carite. Los ingredientes se fundieron a baño maría y una vez que se formó una mezcla se incorporaron $5 \mathrm{~mL}$ de la disolución con $1 \mathrm{~g} / \mathrm{L}$ de mucílago previamente preparada. Se mezcló todo hasta obtener una consistencia viscosa y homogénea.

Luego se preparó una muestra que consistió en una delgada capa homogénea de la crema entre dos portaobjetos. El espesor de esta muestra de crema protectora fue de $0.04 \mathrm{~mm}$. Luego se midió la absorbancia de las muestras empleando un espectrofotómetro. El factor de protección solar de la disolución y la crema se determinaron siguiendo la metodología in vitro descrita por Mansur et al. 1986. Este análisis consiste en un método espectrofotométrico en el cual la formulación se diluye hasta una concentración de $0.2 \mathrm{mg} / \mathrm{ml}$, condición establecida para crear una correlación con el método in vivo.

RAMÍREZ-GRANADOS, Juan Carlos, DÍAZ-PÉREZ, César, ALMANZA-GUERRERO, Lisbeth y ENRÍQUEZ-ARREDONDO, María José. Uso no-convencional del mucílago de avena en fotoprotección. Revista del Diseño Innovativo. 2019 
A través de la ecuación propuesta por Mansur (Ec. 4) se evaluó el FPS en el rango de 290 a $320 \mathrm{~nm}$ (rango UVB) con intervalos de 5 nm.

$$
F P S=F C \sum_{290}^{320} E E(\lambda) I(\lambda) A b s(\lambda)
$$

Donde: FPS es el factor de protección solar, $F C$ es un factor de corrección con valor de $10, E E(\lambda)$ es el efecto eritemogénico de la radiación de longitud de onda $\lambda, I(\lambda)$ es la intensidad del Sol en la longitud de onda $\lambda$, $A b s(\lambda)$ es la absorbancia de la solución en la longitud de onda $\lambda$. Las mediciones fueron realizadas por triplicado y promediadas.

EL producto del efecto eritemogénico y la intensidad de la radiación en cada longitud de onda $E E(\lambda) I(\lambda)$ es una constante que fue determinada por Sayre et al. (Tabla 1).

\begin{tabular}{|c|l|l|l|l|l|l|l|}
$\lambda$ & 290 & 295 & 300 & 305 & 310 & 315 & 320 \\
$E \mathrm{E} \times I$ & 0.015 & 0.082 & 0.287 & 0.328 & 0.186 & 0.084 & 0.018 \\
\hline
\end{tabular}

Tabla 1 Producto del efecto eritemogénico $E E(\lambda)$ y la intensidad de la radiación $I(\lambda)$ entre 290 y $320 \mathrm{~nm}$

Fuente: Sayre R, Desrochers D, Marlow E

\section{Resultados}

A continuación, se muestran las absorbancias espectrales y resultados del FPS obtenido para las muestras.

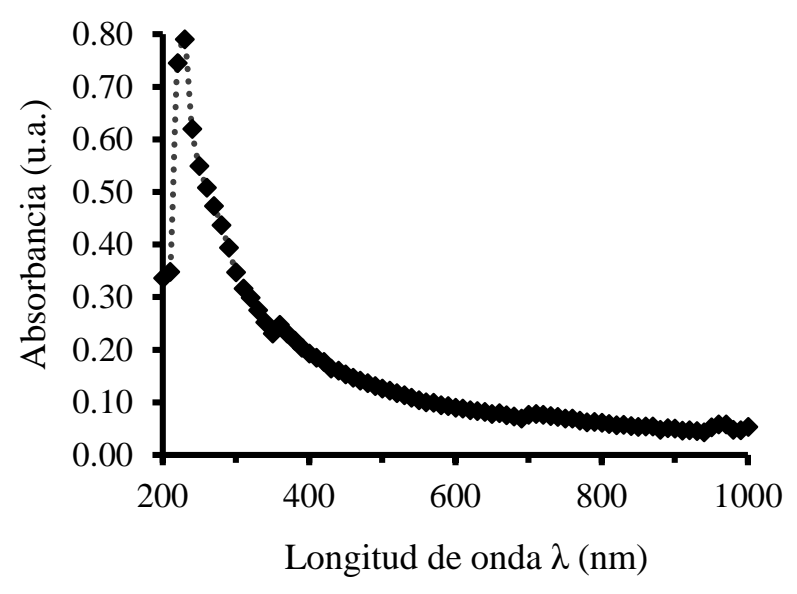

Gráfico 1 Espectro de absorbancia de una disolución con $1 \mathrm{~g} / \mathrm{L}$ de mucílago de avena

En el Gráfico 1 se muestran la absorbancia espectral de una disolución con mucílago de avena en las regiones UV-VisibleIR Cercano. Aquí se observa un perfil con una fuerte banda de absorción cuyo valor pico se encuentra cercano a $230 \mathrm{~nm}$.
La absorbancia de la disolución con mucílago de avena disminuye conforme se incrementa la longitud de onda desde los $230 \mathrm{~nm}$ hasta los $1000 \mathrm{~nm}$. El ancho completo a la mitad del máximo de esta banda va al menos de los 220 a los $290 \mathrm{~nm}$. Por lo tanto, esta banda tiene su máxima absorción en la región UVC, aunque el mucílago de avena también presenta absorción de radiación UVB y UVA. Esta característica del mucílago puede implicar la necesidad de combinar varios ingredientes activos en un filtro solar para cubrir un espectro radiante más amplio.

El Gráfico 2 muestra la absorbancia espectral de una alícuota de avena obtenida antes de la etapa de precipitación con etanol y en donde había una alta concentración de mucílago. En esta alícuota, la absorbancia del mucílago presenta un perfil muy similar al del Gráfico 1 . Aquí, el mucílago también presenta una banda de absorbancia con valor pico centrado en 230 $\mathrm{nm}$. El ancho completo a la mitad del máximo de esta banda también va de los 220 a los $290 \mathrm{~nm}$. Lo cual indica que esta alícuota sólo tiene mucílago disuelto y con una alta concentración.

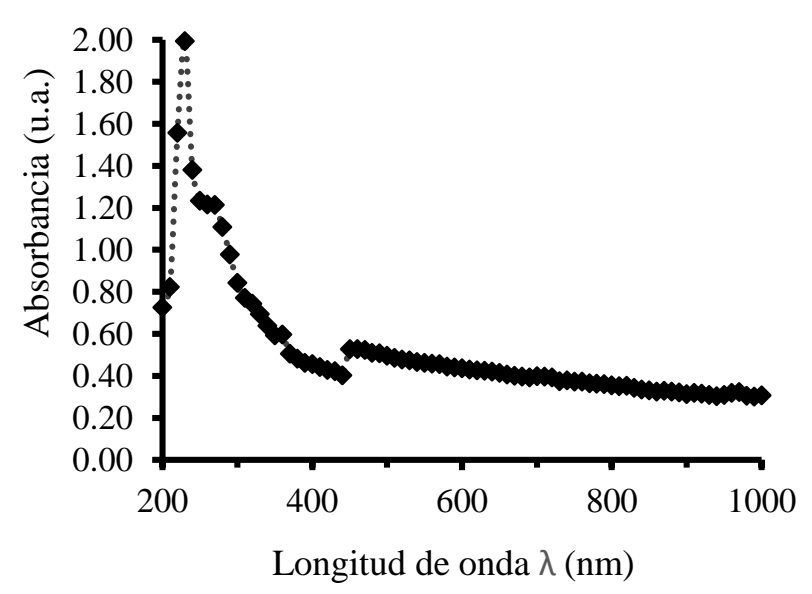

Gráfico 2 Espectro de absorbancia de la alícuota con mucílago de avena

A partir de los espectros de absorbancia mostrados en los Gráficos 1 y 2 se determinó la longitud de onda a la que el mucílago presenta su mayor absorbancia $\left(\lambda_{\max }\right)$. Dicho valor de $\lambda_{\max }$ se utilizó para determinar el coeficiente de extinción $(\varepsilon)$ del mucílago de avena empleando la ecuación de Lambert-Beer (Ec. 2), y así tener un indicio de la cantidad de luz absorbida por este material orgánico. Luego se obtuvo la curva de la calibración para el mucílago de avena. Esta curva de calibración fue obtenida midiendo la absorbancia de una serie de soluciones con concentraciones conocidas de mucílago a 230 $\mathrm{nm}$.

RAMÍREZ-GRANADOS, Juan Carlos, DÍAZ-PÉREZ, César, ALMANZA-GUERRERO, Lisbeth y ENRÍQUEZ-ARREDONDO, María José. Uso no-convencional del mucílago de avena en fotoprotección. Revista del Diseño Innovativo. 2019 


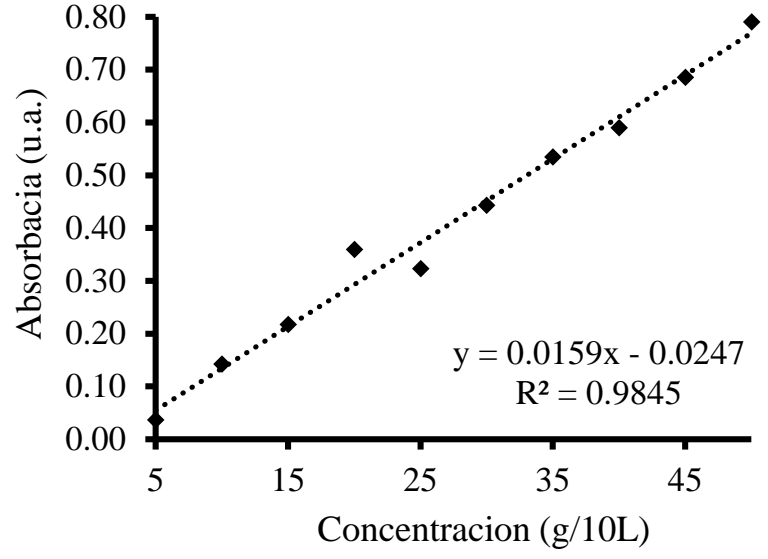

Gráfico 3 Curva de calibración para el mucílago de avena a $230 \mathrm{~nm}$.

En el Gráfico 3 se observa que la absorbancia es directamente proporcional a la concentración del mucílago en disolución acuosa dentro del rango de 0.5 a $5.0 \mathrm{~g} / \mathrm{L}$. Este comportamiento lineal indica que la Ley de Lambert-Beer se cumple para disoluciones con este rango de concentraciones. En disoluciones con concentraciones de mucílago mayores que $5.0 \mathrm{~g} / \mathrm{L}$ se pierde la linealidad y se generan desviaciones negativas. Con una regresión lineal se obtuvo la ecuación de la recta que mejor ajusta a las mediciones. El valor de la pendiente de la recta de ajuste fue de 0.0159; por lo tanto, el coeficiente de extinción es de $0.159 \mathrm{~L} /(\mathrm{g}-\mathrm{cm})$.

Las mediciones espectrofotométricas son parte de la metodología para la determinación del FPS in vitro descrita por Sayre (1979) y utilizada por Mansur et al. (1986) para obtener una buena correlación con pruebas in vivo con la misma finalidad para productos con filtros orgánicos, incluyendo al mucílago de avena. Las mediciones obtenidas fueron utilizadas en la ecuación Ec. 4 para el cálculo de FPS. En la Tabla 2 se muestran las absorbancias empleadas en dicha ecuación para una disolución con 0.2 g/L de mucílago de avena, lo que da un FPS de 4.245 .

\begin{tabular}{|r|l|l|r|}
\multicolumn{1}{r|}{$\lambda(\mathrm{nm})$} & Abs & EE $\times \mathrm{I}$ & EE $\times \mathrm{I} \times$ Abs \\
\hline 290 & 0.489 & 0.015 & 0.007 \\
\hline 295 & 0.467 & 0.081 & 0.038 \\
\hline 300 & 0.444 & 0.287 & 0.127 \\
\hline 305 & 0.419 & 0.327 & 0.137 \\
\hline 310 & 0.402 & 0.186 & 0.074 \\
\hline 315 & 0.386 & 0.083 & 0.032 \\
\hline 320 & 0.377 & 0.018 & 0.006 \\
\hline & & $\boldsymbol{F P S}$ & $\mathbf{4 . 2 4 5}$ \\
\cline { 3 - 4 } & & &
\end{tabular}

Tabla 2 Absorbancia $(A b s)$ de una disolución con $0.2 \mathrm{~g} / \mathrm{L}$ de mucílago de avena y sus respectivas constantes de Sayre para el cálculo del FPS
Por su parte, en la Tabla 3 se muestran las absorbancias y constantes de Sayre empleadas en la Ec. 4 para la crema con mucílago de avena. También se muestra el resultado del FPS que fue de 11.157.

\begin{tabular}{|c|c|c|c|}
\hline$\lambda(\mathrm{nm})$ & Abs & $\mathrm{EE} \times \mathrm{I}$ & $\mathrm{EE} \times \mathrm{I} \times \mathrm{Abs}$ \\
\hline 290 & 0.825 & 0.015 & 0.012 \\
\hline 295 & 0.966 & 0.081 & 0.078 \\
\hline 300 & 1.113 & 0.287 & 0.319 \\
\hline 305 & 1.187 & 0.327 & 0.389 \\
\hline 310 & 1.092 & 0.186 & 0.203 \\
\hline 315 & 1.102 & 0.083 & 0.092 \\
\hline 320 & 1.081 & 0.018 & 0.019 \\
\hline & & FPS & 11.157 \\
\hline
\end{tabular}

Tabla 3Absorbancia $(A b s)$ de una crema con mucílago de avena y su respectiva constante de Sayre $E E \times I$ para el cálculo del FPS

Según la clasificación de la Tabla 4, la capacidad fotoprotectora in vitro del mucílago de avena por si sólo es baja; sin embargo, cuando se mezcla con otros ingredientes que son parte de la formulación de la crema base adquiere una capacidad fotoprotección media.

\begin{tabular}{|l|c|}
\cline { 2 - 2 } \begin{tabular}{|l} 
Capacidad \\
fotoprotectora in vitro
\end{tabular} & $\begin{array}{c}\text { Clasificación } \\
2<F P S<6 \\
\text { protección baja }\end{array}$ \\
\cline { 2 - 2 } & $\begin{array}{c}8<F P S<12 \\
\text { protección media }\end{array}$ \\
\cline { 2 - 2 } & $\begin{array}{c}15<F P S<25 \\
\text { protección alta }\end{array}$ \\
\cline { 2 - 2 } & $\begin{array}{c}30<F P S<50 \\
\text { protección muy alta }\end{array}$ \\
\cline { 2 - 2 } & $\begin{array}{c}F P S>50 \\
\text { protección ultra alta }\end{array}$ \\
\hline
\end{tabular}

Tabla 4 Evaluación de la capacidad fotoprotectora in vitro de las sustancias según Mansur et al. (1986)

También es importante considerar que la capacidad de fotoprotección del mucílago es dependiente de su concentración en la disolución o en la crema; por lo tanto, la capacidad fotoprotectora de la crema aumentará si se incrementa la concentración del mucílago.

\section{Discusiones y conclusiones}

Los mucílagos pueden ser empleados como ingredientes activos para la absorción de radiación UV en protectores solares orgánicos para la piel. Existen diversos estudios acerca de mucílagos que confirman que son una alternativa viable para elaborar protectores solares orgánicos atóxicos para las personas y el ambiente. 
Aquí se estudió la absorbancia espectral del mucílago de avena para determinar si es factible usarlo como ingrediente activo en protectores solares orgánicos para la piel. Para este propósito se prepararon soluciones acuosas con diferentes concentraciones de mucílago de avena y las analizamos con un espectrofotómetro en el rango de 200 a $1000 \mathrm{~nm}$ para obtener la absorbancia espectral del mucílago. Se encontró que el mucílago de avena tiene una banda de absorbancia con valor pico en la región UVC y que se extiende a las regiones UVA y UVB que constituyen radiación ionizante que puede dañar la piel.

Los resultados de la determinación del FPS de la crema protectora indican que dadas las propiedades del mucílago de avena sería posible elaborar filtros solares con este material orgánico como ingrediente activo por su absorción de radiación UV, inocuidad y capacidad para retener humedad, especialmente en combinación con otros ingredientes naturales que mejoren su capacidad de fotoprotección a fin de ampliar su espectro de absorbancia a las regiones UVB y UVA.

También será útil incrementar la concentración del mucílago en la formulación para mejorar su capacidad de fotoprotección e incrementar su FPS. Los resultados indican que, si una formulación tiene la concentración adecuada de mucílago de avena, entonces ésta puede llegar a absorber radiación UV de manera similar a un protector solar químico. Esto confirma que un protector solar elaborado con ingredientes orgánicos puede tener un desempeño equiparable al de los protectores químicos, pero con la ventaja de ser más benéfico para los consumidores por su inocuidad, capacidad para hidratar la piel durante más tiempo y retención de humedad; además de ser amigable con el medio ambiente. Se requiere más investigación para optimizar la capacidad fotoprotectora de los productos adicionados con mucílago de avena y para estudiar sus aspectos cosméticos.

\section{Agradecimientos}

Este trabajo fue realizado con el apoyo 699DIAG de la Rectoría del Campus CelayaSalvatierra y la División de Ciencias de la Salud e Ingenierías de la Universidad de Guanajuato. También se agradece a Dayana Sarahi Bedolla Ortiz por su valioso apoyo.

\section{Referencias}

Castanedo, J.; Martínez, k.; Hernández, D. \&, Valdez, G. (2014). Evaluación in vitro de filtros solares comerciales disponibles en América Latina. Invest Clin 55; 142 - 154.

González-Púmariega, M.; Tamayo, M. V. \& Sánchez-Lamar, A. (2009). La radiación ultravioleta: Su efecto dañino y consecuencias para la salud humana. Theoria 18: 69-80.

Hellwig, R.; Gripentrog, M. \& Templeton, S. (2012). Shining the Light on Sunscreen. US Pharm 37(4):36-39.

Latha, M. S.; Martis, J.; Shobha, V.; Sham Shinde, R.; Bangera, S.; Krishnankutty, B.; Bellary, S.; Varughese, S.; Rao, P. \& Naveen Kumar, B. R. (2013). Sunscreening agents: A Review. Journal of Clinical and Aesthetic Dermatology 6: 16-26.

Rai, R.; Shanmuga, S. C. \& Srinivas, C. R. (2012). Update on photoprotection. Indian $J$ Dermatol 2012; 57: 335-42.

Parhi, R. (2019). The Role of Phytocompounds in Cosmeceutical Applications. Medicinal Plants: Chemistry, Pharmacology, and Therapeutic Applications, 139.

Pereira, G.; Bizari, M. \& Cebim, M. (2019). Estudio de la estabilidad coloidal y propiedades ópticas de las cremas de protección solar. Redalyc. Recuperado de http://www.redalyc. org/jatsRepo/429/42958876005/index.html.

Prakash, D., \& Gupta, C. (2019). Phytopharmaceutical applications of nutraceutical and functional foods. In Complementary and Alternative Medicine: Breakthroughs in Research and Practice (pp. 182-204). IGI Global.

Sánchez Saldaña, L.; Lanchipa Yokota, P.; Pancorbo Mendoza, J.; Regis Roggero, A. \& Saenz Anduaga, E. M. (2002). Fotoprotectores tópicos. Revista Peruana de Dermatología 12:156-163.

Singh, P. \& Nanda, A. (2014). Enhanced sun protection of nano-sized metal oxide particles over conventional metal oxide particles: An in vitro comparative study. International Journal of Cosmetic Science 36: 273-283. 